\title{
Zur Genese von Badlands in Bolivien
}

\section{Zusammenfassung}

Badlands liegen auf dem bolivianischen Altiplano und in den gegen E gerichteten Andendurchbruchstälern. Ihre klimatischen Voraussetzungen und ihr Formenschatz werden diskutiert. Für geomorphologische Prozesse ist die Regenzeit entscheidend, wobei der intensiven Verdunstung Bedeutung zukommt. Überflutung und sofortige Austrocknung wechseln kurzfristig, was allgemein für Tropen typische Kleinformen hervorbringt. Dazu zählen Klein-Canyons, Erdpyramiden, (Quell-) Nischen, Gefällsknicke, Racheln, Kämme und als besonders behandelte Formen Mäander und «strauchbesetzte Deflationskuppen».

\section{Resumen}

Hay bad-lands (tierras malas) en el Altiplano boliviano y en los valles mesotérmicos. Se discuten sus condiciones climáticas y los procesos que forman su superficie. La época de lluvias causa la mayoría de las formas geomórficas, durante la cual la evaporación es también intensa. Inundaciones y resecación rápida oscilan en tiempo breve, lo que causa formas pequeñas generalmente típicas para regiones tropicales. Mencionamos pequeños cañones, pirámides de tierra, cabeceras de valle poco espaciadas, terrazas, regueros, barrancos (huaicos), y tratamos especialmente meandros y morros formados por deflación eólica.

\section{Untersuchungsobjekt}

In Bolivien sind viele Fundstellen von Säugetierfossilien bekannt geworden (z. B. bei Tarija nach AHLFELD 1946, S. 310 f.). Es handelt sich meistens um Gebiete ausgedehnter mergeliger Pliozän- oder Pleistozänsedimente, die durch intensive Abtragungsprozesse in Badlands verwandelt worden sind. Während gemeinsamen Exkursionen mit Paläontologen hatte ich 1973-1975 Gelegenheit, dazu geomorphologische Untersuchungen im nördlichen Teil des bolivianischen

\footnotetext{
1) In den Jahren 1973-1975 weilte ich mit Unterstützung des Schweizerischen Nationalfonds in Bolivien.

2) s. dazu MONHEIM (1956, S. 34 und 58 f.) zu Niederschlägen und Trockengrenze im nördlichen Altiplano.
}

Altiplano anzustellen1). Unter Badlands seien unwegsame, zerschrundete Gelände verstanden, die nur eine schüttere Vegetation aufkommen lassen und in zahllose unbeständige Formen der Erosion und Denudation zerlegt sind. Im folgenden wird versucht, das Altiplano im Bereich des Departements La Paz klimatisch zu charakterisieren und daraus Folgerungen auf die Abtragungsprozesse in Badlands zu ziehen. Das Untersuchungsgebiet erstreckt sich vom Titicacasee (ca. ab $15^{\circ} 30^{\prime} \mathrm{S}$ ) bis fast $18^{\circ} \mathrm{S}$ und liegt im allgemeinen auf Höhen von $3800-4200 \mathrm{~m}$ ü. M. Es entstand hauptsächlich im Pliozän als Depression zwischen der West- und Zentralkordillere und war im Pleistozän nur spärlich vergletschert. Die Hauptentwässerung erfolgt im W durch den río Desaguadero, im E sind dessen Zuflüsse río Colorado und río Kheto durch eine Talwasserscheide bei Calamarca getrennt. Östlich dieses abflußlosen Entwässerungssystems des Hochlandes befinden sich mehrere aride Täler. Diese «Valles» wurzeln im Altiplano, fallen aber abrupt in Badlands ab und öffnen sich als Windpforten zum Amazonasbecken hin.

\section{Klimatische Besonderheiten}

Das Klima des nördlichen Altiplano ist durch den Wechsel von Feucht- und Trockenzeit geprägt. Die sommerliche Regenzeit von November bis März bewirkt häufig katastrophale Rutschungen in Siedlungen und bei schlecht angelegten Straßen. Da dann allgemein die Abtragungsprozesse am intensivsten sind, betrifft unser Überblick zum Klima hauptsächlich die Regenzeit.

Fig. 1 zeigt die Lage von 15 bolivianischen Klimastationen, die 1961-1973 einigermaßen regelmäßig arbeiteten. In Fig. 2 ist dazu die Niederschlagssumme ihres feuchtesten Monats (meistens Januar) in Relation zur Mitteltemperatur des wärmsten Monats (meistens auch Januar) gesetzt. Es fällt auf, daß der südöstliche Abschnitt im Höhepunkt der Regenzeit relativ warm und trocken ist. Dieses Gebiet häufiger Badlands weist also auch regenzeitlich eine starke Verdunstung auf2). Genaue Meßreihen zur Verdunstung sind in Bolivien spärlich. Bei Fig. 3 wurde meist auf Klima-

Dr. Kurt Graf, Oberassistent am Geographischen Institut der Universität Zürich, Blümlisalpstrasse 10, 8006 Zürich. 
Fig. 1: Klimastationen auf dem Altiplano des Departements La Paz zwischen 3800 und 4100 m ü. M. sowie La Paz/ San Calixto, $3660 \mathrm{~m}$ ü. M.

Fig. 2: Die Klimastationen von Fig. 1, nach Niederschlag und Temperatur im Januar geordnet (genauer: höchste Werte des Monatsniederschlags und der Monatsmitteltemperatur wurden gewählt). Die Orte mit dem trockensten und wärmsten Klima stehen rechts unten bzw. bei Fig. $1 \mathrm{im} \mathrm{SE}$ Quelle: Anuarios Meteorológicos 1961-1973.

jahrbücher (Anuarios Meteorológicos) 1960-1972 zurückgegriffen. Für Copacabana erwähnt ANTEZANA (1958, S. 186) Daten zur maximalen Verdunstung auf dicht krautbesetzter Oberfläche (= potentielle Evapotranspiration). Eine zehnjährige Beobachtungszeit gibt er als Grundlage an, ohne sie näher zu bezeichnen. Messungen mit Evapotranspirometern ergeben gesamthaft zu kleine Verdunstungswerte auf dem Altiplano, da ein dichtes Pflanzenkleid mit seiner relativ großen Rückstrahlung des Sonnenlichts (Albedo) weitgehend fehlt. Anderseits geben Piche-Evaporimeter mit ihrer kleinen Test-Wasserfläche $\left(13 \mathrm{~cm}^{2}\right.$ nach THORNTHWAITE 1955, S. 15) allgemein zu hohe Verdunstungswerte, da die meiste Energie zur Evaporation von der Luft der Umgebung stammt. Für Desaguadero entnahm ich solche Daten zur maximalen Verdunstung auf Wasserflächen (= potentielle Evaporation) bei KESSLER (1970, S. 343), der sie als Mittelwert der Küstenstationen Desaguadero und Capachica/Peru angibt. In La Paz und Patacamaya wurde durch den Servicio Nacional de Meteorología mit Verdunstungswaagen gemessen, die mit einem Durchmesser der Wasserfläche von ca. $1.20 \mathrm{~m}$ genaue Werte der potentiellen Evaporation geben1).

In Badlands (z. B. von Patacamaya) bleiben die Monatsniederschläge stets deutlich hinter den maximal möglichen Verdunstungswerten zurück, es herrscht offenbar eine besonders intensive Verdunstung. Ein Vergleich der Differenz von Niederschlag und Verdunstung ist für unser Untersuchungsobjekt indessen nicht ganz stichhaltig, weil

1. die wirkliche Verdunstung (= aktuelle Evaporation) auf sandig-lehmigem Untergrund nicht jener über Wasserflächen (= potentielle Evaporation) gleichkommt.

2. die Niederschläge unregelmäßig fallen und kurzfristige Überschwemmungen bzw. Übersättigung hervorrufen können.

3. die Wasserbilanz auch vom Abfluß gesteuert wird und damit bei zu kleiner Verdunstung trotzdem zu vollständiger Austrocknung führen kann2).

1) s. dazu die Vergleiche mit zwei verschieden berechneten Werten der potentiellen Evaporation in Puno/Peru von KESSLER (1970, S. 246).

2) KESSLER (1970, S. 251) läßt bei seiner Klimauntersuchung des Titicaca-Beckens den $\mathrm{Zu}$ - und $A b f l u B$ auch unberücksichtigt und behandelt die «klimatische Wasserbilanz» (= Niederschlag minus Verdunstung).
Fig. 3: Klimadaten zu vier ausgewählten Stationen: Copacabana (3840 m ü. M.) und Desaguadero (3810 m) am Titicacasee, Patacamaya $(3790 \mathrm{~m})$ und La Paz (Colegio San Calixto 3660 m, das im Gegensatz zum 4100 m hohen El Alto von La Paz im Talkessel unten liegt). In den Darstellungen zur klimatischen Wasserbilanz (s. Anm. 4) sind die Monatsmittel der potentiellen Verdunstung strichpunktiert aufgetragen, jene der Niederschläge mit ausgezogenen Linien. Die Rasterflächen zeigen ein Überwiegen des Niederschlags an. Quellen siehe Text.
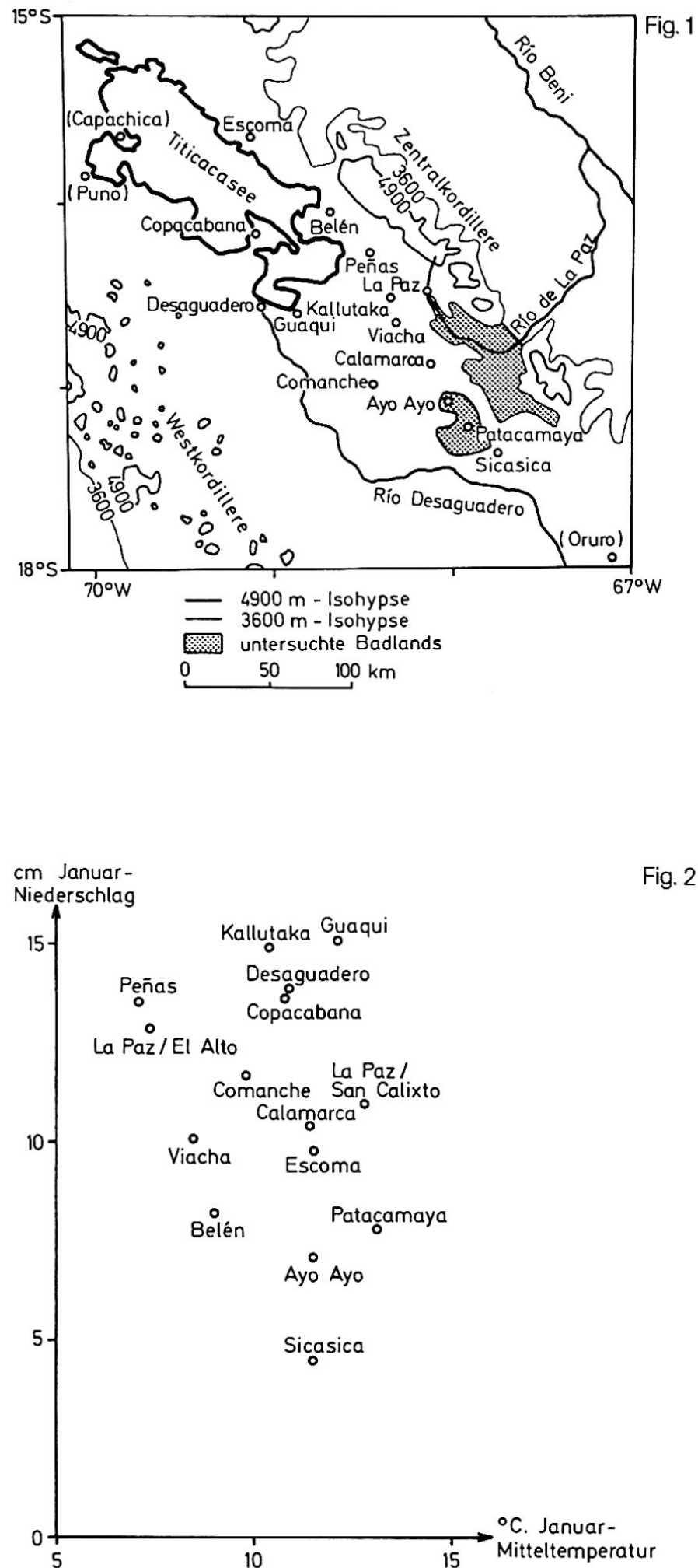
Fig. 3 Copacabana

Desaguadero

Mitteltemperatur 1960-68

Mitteltemperatur 1966-70
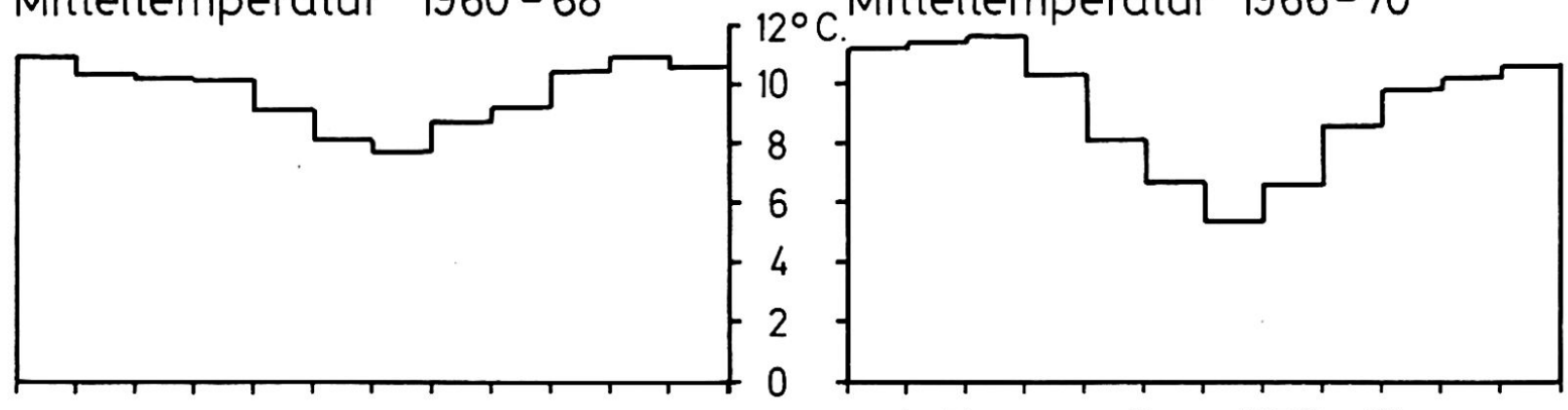

potentielle Evapotranspiration

Niederschläge 1960-68

pot. Evaporation 1958-61

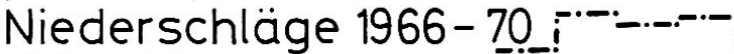
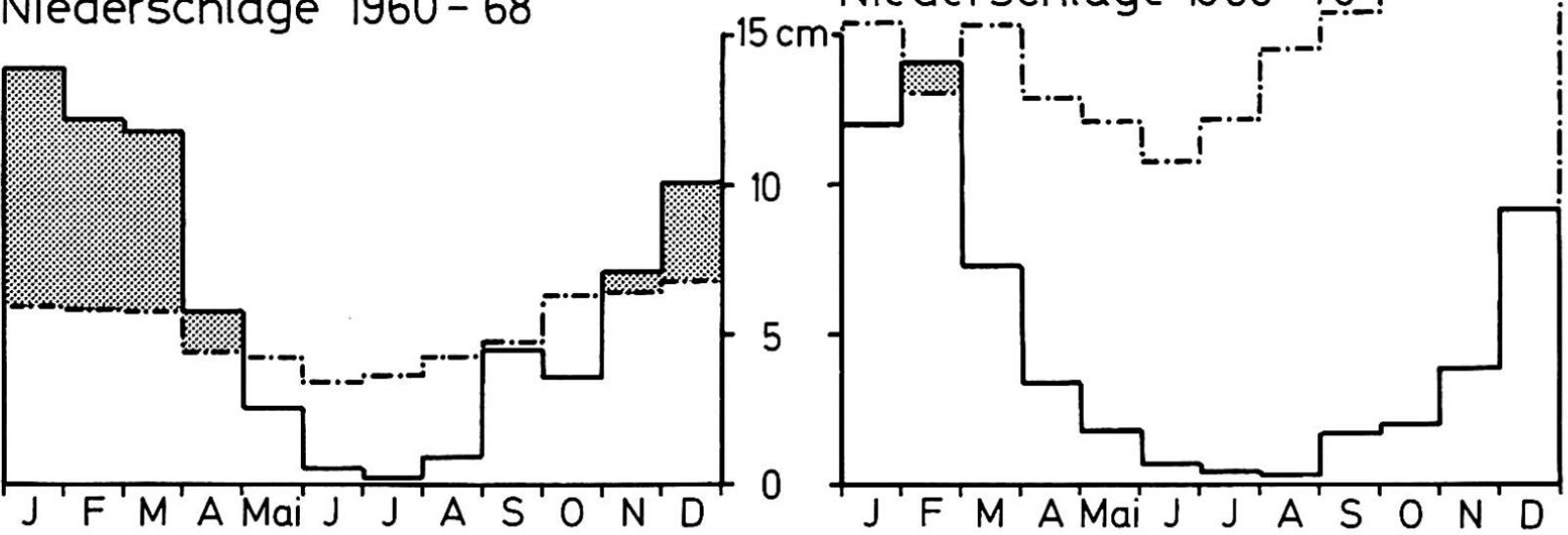

La Paz / San Calixto

Mitteltemperatur 1961-70

\section{Patacamaya}

Mitteltemperatur 1962-71
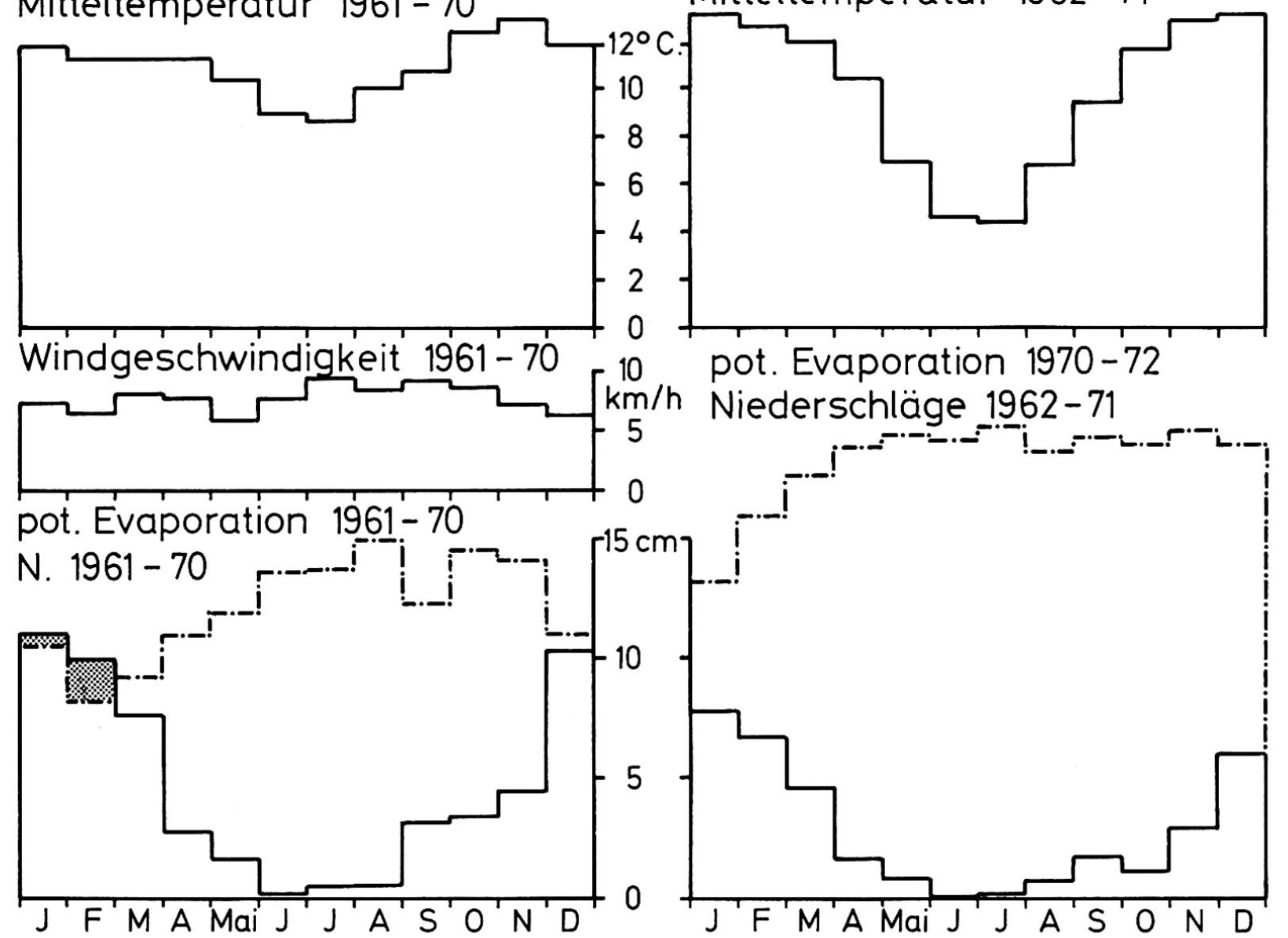
In La Paz zeichnet sich ungefähr ein Gleichgewicht in den Regenzeitmonaten ab. Man kann folgern, daß auf $3660 \mathrm{~m}$ in La Paz vom Klima her noch keine ausgeprägten Badlands erwartet werden dürfen. Sie setzen auch wirklich erst $300 \mathrm{~m}$ weiter unten, im Mondtal, weitflächig ein (Abb.1). Damit erklärt sich bei Fig. 2, warum La Paz/San Calixto und v. a. La $\mathrm{Paz} / \mathrm{El}$ Alto überraschend weit oben zu liegen kommen. Höhenwärts wird nämlich das Klima in $\mathrm{La} \mathrm{Paz}$ stetig feuchter, dem río de La Paz abwärts folgend, erreicht man schnell extrem aride Badlands und Kakteenlandschaften. Leider fehlen für solche Valles Klimadaten, aber sie lägen in Fig. 2 deutlich rechts unten. Für die Verdunstung wirkt sich überdies der tageszeitliche Wind aus (TROLL 1968, S.49), der im Mondtal unten mindestens so stark wie im Stadtzentrum von La Paz (San Calixto) weht. In Fig. 3 sind diese Daten zur Windgeschwindigkeit als Monatsmittel angegeben. Sie sind ungeachtet der Windrichtung gemittelt worden, weil in unserer Untersuchung allein die Stärke des Windes interessiert. Gute Ventilation beschleunigt die Verdunstung bzw. Austrocknung der Oberfläche und deren Bereitstellung für den Abtrag. Die stärksten Winde wehen in La Paz während der winterlichen Trockenzeit, sie sind aber während des ganzen Jahres beachtlich.

Ebenfalls nur mit Vorbehalt dürfen die Monatsmitteltemperaturen im Hinblick auf Austrocknung, Verwitterung und Abtrag hin beurteilt werden. In $\mathrm{La} \mathrm{Paz}$ zeigten beispielsweise Stichproben im Oktober 1974, $\mathrm{da} ß$ die Mittagstemperaturen am Schatten $18-20^{\circ} \mathrm{C}$ betragen können gegenüber $40-45^{\circ} \mathrm{C}$ an der Sonne. Bei so hohen Temperaturen erfolgt die Verdunstung des Oberflächenwassers tagsüber viel schneller, als man aufgrund der Schattentemperaturen erwarten würde. Die effektiven Verhältnisse der Austrocknung sind aber zudem wohl stets durch Lokalfaktoren geprägt. So beschleunigt z. B. der schnelle Abfluß als Sturzbäche in die ariden Valles hinunter die Wiederaustrocknung, was in La Paz die Badlandsbildung edaphisch begünstigt. Die Verdunstung ist überdies in Wirklichkeit ein langsames Einstellen auf das schüttere Pflanzenkleid. Sie stellt für die Landwirtschaft auf dem Altiplano einen Teufelskreis dar: Die primär große Verdunstung bewirkt einen Rückgang der Vegetation, wodurch die Albedo kleiner wird und damit die aktuelle Verdunstung noch größer. Der Trockenheit angepaßte Pflanzen gebieten diesem $\mathrm{Kreis}$ in gewissem Maß Einhalt.

\section{Mechanismus der Abtragungsprozesse}

Mergelige Sedimente bilden eine erste Voraussetzung für Badlands, wie sie beispielsweise auch in allen Breitenlagen der USA vorkommen (Grand Canyon in Arizona, die Regionen Mauvaises Terres und Bad Lands in Dakota). Ausreichendes Gefälle für oberflächlichen raschen Abfluß ist ebenfalls förderlich. Dazu kommen aber in den Randtropen klimatische Gegebenheiten, welche den Abtrag sehr begünstigen. Unser Vergleich von wechselfeuchten Stationen Boliviens ergab, daß gegen $S$ und im Abstieg in die Valles die Trockenheit zunimmt und Badlands sich häufen. Wahrscheinlich ist aber nicht die Aridität an sich für die intensiven Abtragungsvorgänge entscheidend, sondern die Regenzeit. Während ca. fünf Monaten findet die hauptsächliche Abspülung statt. Die Luft bleibt auch während der Regenzeit sehr trocken. Sie bedingt also auch dann noch eine recht wirksame Verdunstung, welche überdies durch die Strahlung des steil einfallenden Sonnenlichts verstärkt wird. Auch in der Regenzeit trocknet daher die Oberfläche zwischen zwei Regenfällen sofort wieder aus (s. auch MONHEIM 1956, S. 55). Das tonige Material schrumpft leicht zusammen und lockert den Verband. Bei erneuten Schauern quellt das aufbereitete Material und kann abgespült werden. So wiederholt sich in kurzen Abständen das Wechselspiel von Verwitterung und $\mathrm{Ab}$ spülung. Von November bis März pulsiert der Abtragungsmechanismus kurzatmig zwischen trockener und durchnäßter Feinerde, zwischen Aufbereitung und Abtrag. Damit sind die Voraussetzungen für die zahlreichen, fein herausmodellierten Formen in Badlands geschaffen. Die Großformen werden in Kleinformen zerlegt, bestimmen aber weiterhin das Großrelief. Es scheint, daß die Bildung von Kleinformen allgemein für tropische Abtragungsprozesse typisch ist. Damit soll nicht gesagt sein, daß solche Kleinformungen nicht auch in subtropischen und gemäßigten Breiten bei ähnlichem Klima und Witterungsablauf auftreten. Ein analoges Beispiel in tropischen Gebirgen sind die häufigen kurzfristigen Fröste, welche die Abtragungsvorgänge grundlegend bestimmen und zahlreiche Kleinformen von Strukturböden bewirken1). Auch

1) Die Problematik konvergenter Formung in Trockengebieten und
Hochgebirgen behandelt KAISER (1970, S. 149 f.) und gibt dazu zahlreiche andere Autoren an. 
Abb. 1: Badlands im Mondtal unterhalb La Paz $\left(68^{\circ} 5^{\prime} 40^{\prime \prime}\right.$ W/ $16^{\circ} 33^{\prime} 30^{\prime \prime}$ S, 3200 m ü. M.), Blick nach N talaufwärts.

Abb. 2: Strauchbesetzte Deflationskuppen auf einem Rücken nahe Salla, $20 \mathrm{~km}$ nordöstlich von Sicasica $\left(67^{\circ} 37^{\prime} 40^{\prime \prime} \mathrm{W} /\right.$ $17^{\circ} 9^{\prime} 30^{\prime \prime} \mathrm{S}, 3750 \mathrm{~m}$ ü. M.).
Abb. 3: Abrupter Tälchenanfang, als Nische eingetieft in eine Tuffplatte zwischen Ayo Ayo und Patacamaya $\left(67^{\circ} 56^{\prime}\right.$ W $/$ $17^{\circ} 7^{\prime} 30^{\prime \prime}$ S, 3900 m ü. M.), im Hintergrund im SE ein Inselberg.

Abb. 4: Regelmäßige Mäander zwischen Ayo Ayo und Patacamaya (675' $\mathrm{W} / 17^{\circ} 8^{\prime} 30^{\prime \prime}$ S, 3900 m ü. M.), Blick nach NW.
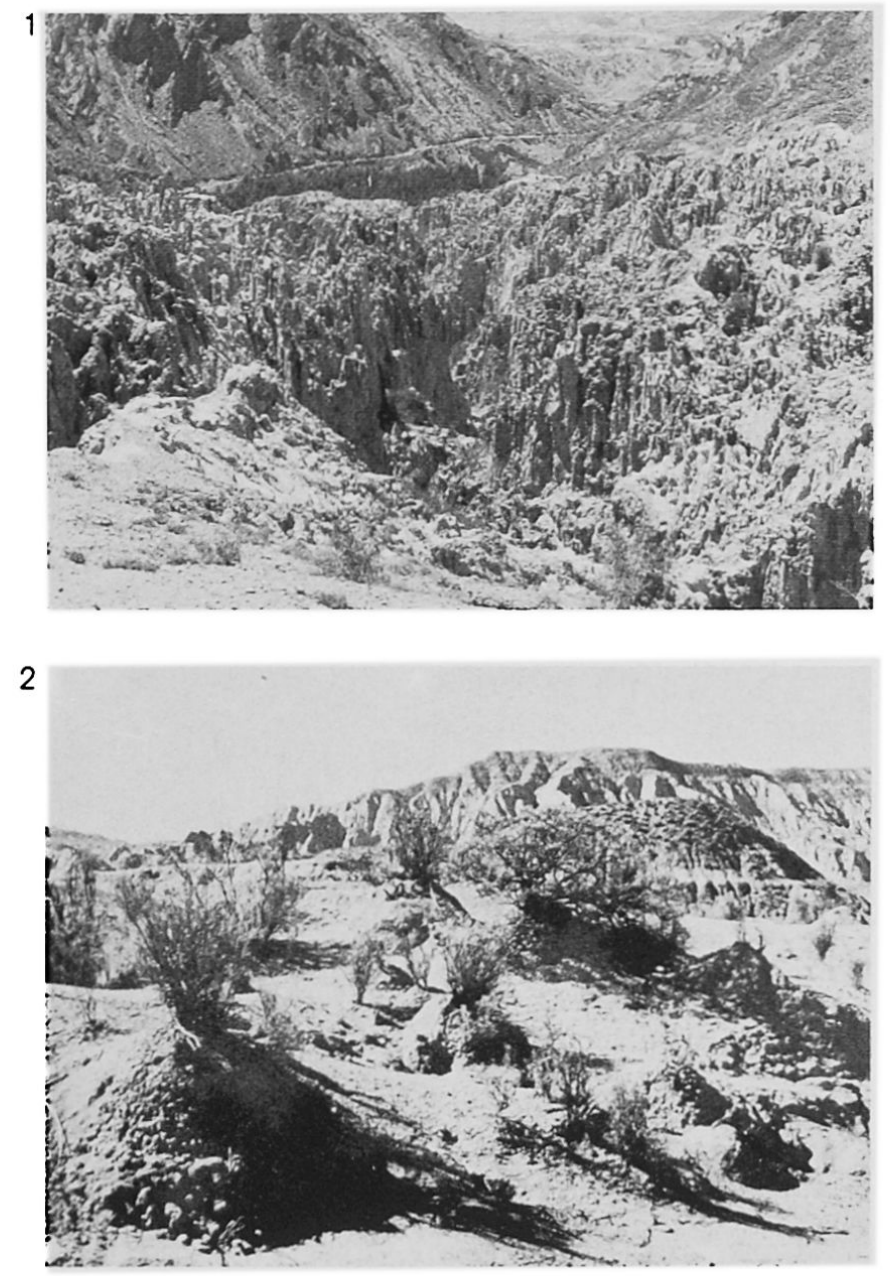

dieser Prozeß ist bis in gemäßigte Breiten sehr wirksam, obschon er äquatorwärts ausgeprägter auftritt.

\section{Oberflächenformen in Badlands}

Die Feldarbeit im unübersichtlichen Gelände von Badlands gestaltet sich oft schwierig, wegkundige Indios haben aber erstaunlichen Orientierungssinn in solchen Labyrinthen. An Großformen findet man Flächenreste, welche eine frühere Verebnung durch Erosion oder Akkumulation nachzeichnen. Es kann sich also dabei einerseits um Inselberge (Tafelberge) handeln, die z. B. südlich von Ayo Ayo von einer harten Tuff-
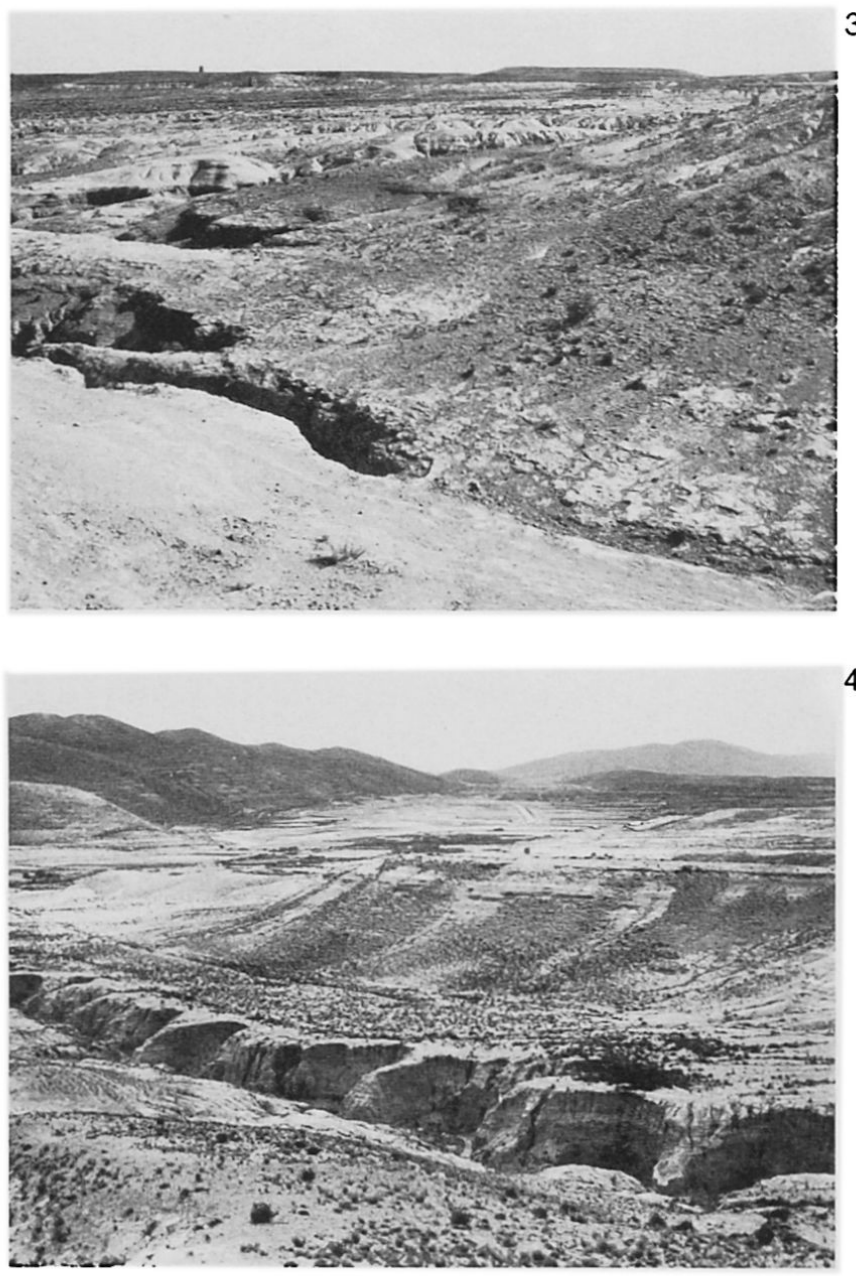

schicht bedeckt sind (HOFFSTETTER 1971, S. 2472). Anderseits sind postglaziale bis rezente Murgänge in den Valles häufig (DOBRovolNy 1962, S. 61 f.). Sie füllen breiartig oft ganze Talabschnitte aus. Als Sammelrinne für die regenzeitlichen Hochwasser dienen breite, schuttbedeckte Talzüge mit steilen Seitenwänden, Canyons.

Das erwähnte Großrelief wiederholt sich in ähnlicher Art in den Kleinformen. Anstelle der Inselberge stehen als Reste häufig Erdpyramiden (Abb. 1). Tälchen mit rechteckigem Canyon-Querprofil können noch bei kleinen Rinnsalen festgestellt werden. Auffällig ist, wie abrupt diese Klein-Canyons mit Nischen auf der ursprünglichen Verebnung beginnen (Abb. 3). Man 
Fig. 4: Grundrißansicht von vier Mäanderschleifen bei der Lokalität von Abb. 4. Die zwei östlicheren zeigen den Normalfall mit scharfer Terrassenkante, die in diesem Beispiel ca. $5 \mathrm{~m}$ zum Wadibett abfällt. Die zwei westlichen sind durch Rutschungen abgeschnürt und weisen Nebenwadis auf.
Fig. 5: Perspektivische Ansicht eines mäandrierenden KleinCanyons südlich von Ayo Ayo $\left(68^{\circ} 1^{\prime} \mathrm{W} / 17^{\circ} 6^{\prime} 30^{\prime \prime} \mathrm{S}\right.$, $3900 \mathrm{~m}$ ü. M.), Blick talaufwärts gegen SE. Der Raster bezeichnet eine harte Schicht ca. $5 \mathrm{~m}$ über der Wadisohle; die unvermittelt in der Pultfläche einsetzenden Nischen von Neben-Wadis sind schwarz markiert.
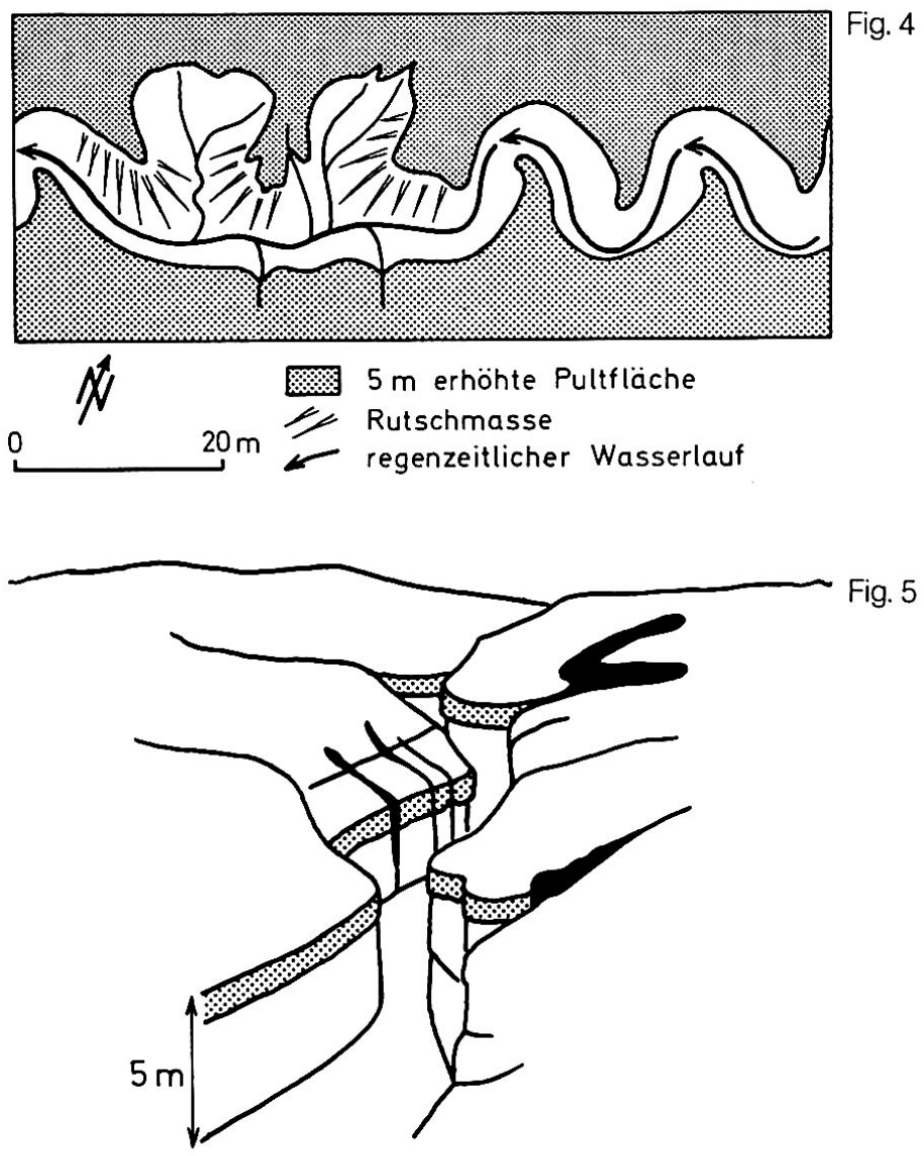

indem er die Verdunstung fördert. In der Regenzeit können Sturzregen den Verband lösen, und im Zuge der sofort anschließenden Austrocknung wird das gelockerte Feinmaterial ausgeblasen.

\section{Literatur}

AHLFELD, F., 1946. Geología de Bolivia. Revista del Museo de La Plata, Rep. Argentina (Nueva Serie), Sección Geología III: 5-370.

ANUARIOS METEOROLOGICOS, 1960-1972, Servicio Nacional de Meteorología e Hidrología, La Paz/Bolivien. anteZANA, O., 1958. Climas de Bolivia según el sistema de Thornthwaite. Rev. de la Fac. de Agronomía (3a época) 34 (2a entrega), La Plata, Argentina: 161-191. BARTELS, G., 1970. Geomorphologische Höhenstufen der Sierra Nevada de Santa Marta (Kolumbien). Giessener Geogr. Schriften 21: $56 \mathrm{~S}$. 
Abb. 5: Das Altiplano Boliviens. Die 3650-4200 m hohe Ebene wird im Westen von zahlreichen Vulkankegeln gesäumt, im Bild von den Payachatas (links Parinacota 6330 m, rechts Pomarapa). Das wechselfeuchte Klima läßt nur eine schüttere Steppenvegetation aufkommen. Große Weideflächen wechseln mit unwegsamen Badlands. Foto K. Graf, 6. Mai 1974.

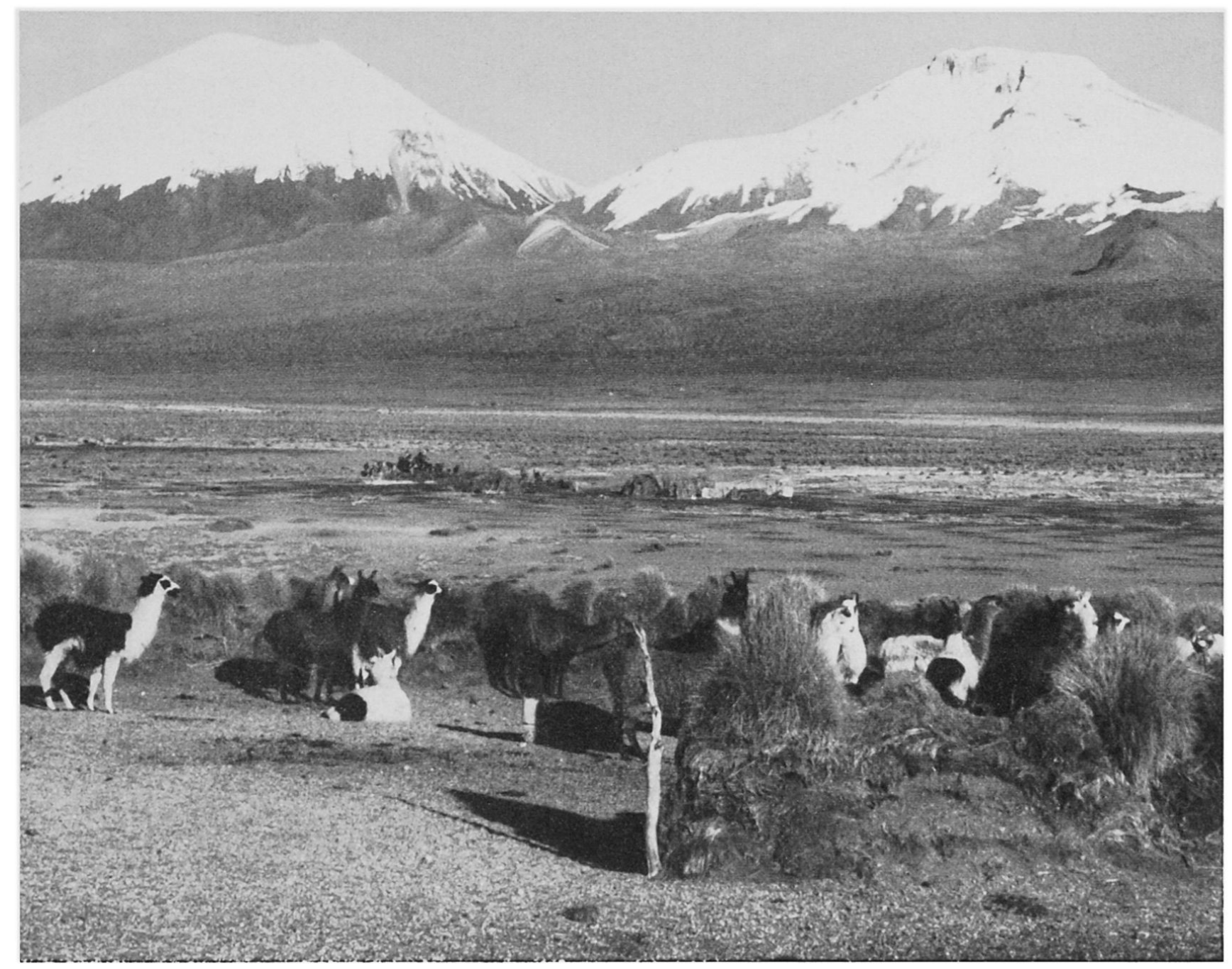

BÜDEL, J., 1971. Das natürliche System der Geomorphologie mit kritischen Gängen zum Formenschatz der Tropen. Würzburger Geogr. Arb. 34: 152 S.

Dobrovolny, E., 1962. Geología del Valle de La Paz. Min. de Minas y Petróleo, Dep. Nac. de Geología, Boletín No. 3 (Especial), La Paz: $153 \mathrm{~S}$.

HOFFSTEtTER, R. et al., 1971. Le gisement d'Ayo Ayo (Bolivie), une succession stratigraphique PliocènePléistocène datée par des Mammifères. Acad. Sc. Paris, t. 273: 2472-2475.

KAISER, K., 1970. Über Konvergenzen arider und «periglazialer» Oberflächenformung. Abh. Geogr. Inst. der Freien Univ. Berlin 13: 147-188.
KESSLER, A., 1970. Über den Jahresgang der potentiellen Verdunstung im Titicaca-Becken. Arch. Met. Geoph. Biokl., Ser. B, 18: 239-252.

MONHEIM, F., 1956. Beiträge zur Klimatologie und Hydrologie des Titicacabeckens. Heidelberger Geogr. Arb. 1: $152 \mathrm{~S}$.

ThornthwaITE, C. W., 1955. The Water Balance. Drexel Inst. of Technology, Publ. in Climatology VIII, 1, Centerton, New Jersey: $104 \mathrm{~S}$.

TROLL, C., 1968. Geo-Ecology of the Mountainous Regions of the Tropical Americas. Colloquium Geogr. Bonn, Bd. 9: 15-56. 